\title{
CSMJ
}

\section{COVID-19 Vaccines in Children and Adolescents}

\author{
(D) Ener Çağrı Dinleyici
}

Eskisehir Osmangazi University Faculty of Medicine, Department of Pediatrics, Eskişehir, Turkey

\author{
ABSTRACT
}

The coronavirus disease-2019 (COVID-19) pandemic has an effect on children, either directly or indirectly. Severe acute respiratory syndrome coronavirus-2 (SARS-CoV-2) immunization in children and adolescents plays a role in containing the COVID-19 pandemic. As of December 2021, a limited number of COVID-19 vaccines have been approved for use in children and/or adolescents. Both mRNA vaccines (BNT162b2 and mRNA 1273) were found to be well tolerated and effective in large phase 2 and phase 3 clinical trials. BNT162b2 vaccine was approved for children and adolescents aged 5 to 18, and mRNA-1273 vaccine for children aged 12 to 17. CoronaVac, an inactivated SARSCoV-2 vaccine, was also found to be safe and immunogenic in a phase $1 / 2$ clinical trial in China, and is presently used for pediatric immunization in some countries as a routine. As COVID-19 is less severe in children than it is in adults, the benefit of its vaccination in children is less than that of adults. Immunization with an effective and safe vaccine in children and adolescents is likely to provide protection against severe COVID-19 infection. Pediatric COVID-19 vaccines may also protect against the long-term effects of COVID-19 (MIS-C and long COVID) and community transmission, as well as mitigate the indirect effects of the pandemic on them. Vaccination should be prioritized for children and adolescents who have an increased risk of severe COVID-19 infection. If vaccines were evenly distributed worldwide, they would be the safest way to return normal life. Otherwise, lowand middle-income countries will crash, resulting in mortality, undermining global recovery, and allowing more virulent variants (such as Omicron) to emerge. If health officials incorporate the COVID-19 vaccine into routine immunization, they should also regularly evaluate their benefits and potential risks.

Keywords: COVID-19, SARS-CoV-2, pandemic, children, vaccine, mRNA vaccine

\section{Introduction}

The coronavirus disease-2019 (COVID-19) pandemic caused by severe acute respiratory syndrome coronavirus-2 (SARS-CoV-2) virus is still ongoing, with recent estimates of more than 270 million cases diagnosed worldwide and more than 5.3 million deaths by the end of 2021 (1). The pandemic primarily affects adults. Adults and the elderly have mortality rates related to COVID 19, and the presence of underlying condition such as cardiovascular disease, diabetes, cancer, chronic lung disease, and immunocompromised condition were linked to these rates (2). While adult patients account for the vast majority of cases and deaths, the pandemic significantly affects children worldwide directly or indirectly $(3,4,5)$.

Address for Correspondence: Ener Çağı Dinleyici Prof. MD, Eskisehir Osmangazi University Faculty of Medicine, Department of Pediatrics, Eskişehir, Turkey

Phone: +90 2222392979 - 2722 E-mail: timboothtr@yahoo.com 0RCID ID: orcid.org/0000-0002-0339-0134 Received: 09.04.2021 Accepted: 12.04.2021

(C) Copyright 2021 by the Cam \& Sakura Medical Journal published by Galenos Publishing House. 
Regarding previous pandemic experiences, a pandemic could only be controlled with widespread use of safe and effective vaccines. Several COVID-19 vaccines have been under study since the first definition of the virus. The first COVID-19 vaccine was created and tested on humans within two months of the publication of the SARS-CoV-2 genomic sequence. There are 168 types of vaccines still being tested in 536 clinical trials in 62 countries worldwide. These vaccines include whole-cell inactivated virus vaccines, mRNA vaccines, DNA vaccines, adenoviral-vectored vaccines, protein-subunit vaccines, and virus-like particle vaccines (6). By December 2021, at least 1 of the 28 COVID-19 vaccines has been approved for emergency use in 194 countries (7). Vaccines are currently available through their full approval, conditional marketing approval, and emergency use authorization (EUA). A majority of these approved vaccines are shown to be effective and safe in adults and were authorized for adult immunization, including increased risk groups such as the elderly, and health-care workers. However, the use of vaccines is limited in children and adolescents. There are currently more than 20 ongoing clinical trials on vaccines in children, including infants of at least 6 months, as well as adolescents $(4,8)$.

\section{COVID-19 in Children and Adolescents}

Children and adolescents are underrepresented in terms of frequency (a small percentage of the total number of COVID-19 cases) and severity when compared to adults since the beginning of the pandemic (4). From December 2019 and October 2021; children under the age of 5 accounted for $2 \%$ of reported global cases and $0.1 \%$ of reported global deaths. Children aged between 5 to 14 years accounted for $7 \%$ of reported global cases and $0.1 \%$ of reported deaths, and adolescents and young adults (15 to 24 years) accounted for $15 \%$ of reported cases and $0.4 \%$ of deaths (9). Despite these results, there were differences in test capacity and other infection control strategies among countries.

The acute infection with SARS-CoV-2 is generally asymptomatic or mild in children and adolescents $(3,4,5)$. It should be noted that children and adolescents are tested less frequently, and cases may go unnoticed (9). Children with mild infection (mainly admitted with fever and/or cough) can be managed safely without hospitalization and they represent the vast majority of pediatric cases $(3,4,5,10)$. A small percentage of children develop severe disease and require hospitalization (only $1.5 \%$ of all COVID-19 hospitalizations) (4). During the recent Delta wave, children had the highest rate of confirmed SARS-CoV-2 infection though hospitalization rates remained low $(11,12)$. Risk factors for severe disease and mortality in children include newborn and young age, obesity, and the presence of underlying conditions such as asthma, congenital cardiac disease, neurological disease, Down syndrome, diabetes mellitus, and cancer $(5,13)$.

The rate admission in the intensive care unit (ICU) ranged from $2 \%$ to $13 \%$; the risk of death from SARS-CoV-2 infection was $0.005 \%$ in children and adolescents, and $0-0.7 \%$ in those hospitalized with COVID-19 (10). In the United States, from March 2020 to June 2021; 26.5\% of hospitalized children and adolescents with COVID-19 were admitted to an ICU, while $6.1 \%$ required invasive mechanical ventilation (14). The involvement of the respiratory system is the leading cause of hospitalization and the need for intensive care. Extrapulmonary system involvement (cardiac and neurological findings) are relatively uncommon and frequently coexist with pulmonary disease (5). Mortality from COVID-19 is extremely low in children, accounting for only $0.08 \%$ of all COVID-19 deaths (4). The majority of evidence on the risk of severe COVID-19 and death in children and adolescents comes from studies in high-resource settings. Mortality rates vary greatly between countries due to factors such as malnutrition, healthcare access, and delayed diagnosis. A recent systematic review demonstrated that the impact of pediatric COVID-19-related fatalities could be greater in low- to middle-income countries than in high-income countries $(3,9)$.

The age distribution of SARS-CoV-2 infection varies according to circulating variants, mitigation strategies and vaccine coverage in the community. During the most recent Delta variant dominant period, 38\% of all cases reported in the United States were children aged 5-11 years (15). More than 8,300 hospitalizations for severe COVID-19 infection in children aged 5 to 11 years were reported and approximately one-third of them required ICU admission (15). As with other respiratory viruses, the primary management priorities in children with COVID-19 are adequate hydration and supportive care. Recommendations for COVID-19 therapy in children (remdesivir, dexamethasone, and tocilizumab) are primarily extrapolated from adult regimens (2).

Children of all ages can become infected and spread the virus to others. The majority of children become infected through contact with an adult. Infection rates in children and adults were comparable whether schools were open or closed. According to secondary attack rates, children were less affected than adolescents and adults $(9,16)$. However, there is little or no information available on the effects of the recent Omicron (B.1.1.529) variant on children and adolescents (17). 
In addition to complicated and uncomplicated acute COVID-19 infection, long-term consequences of SARS-CoV-2 infection could be more of a concern in the pediatric age group (10). Multi-system inflammatory syndrome in children (MIS-C) is characterized by fever, rash, conjunctival injection, gastrointestinal symptoms, and shock due to myocardial dysfunction (3). Children with MIS-C have a history of COVID-19 or an exposure about 4-6 weeks prior to the onset of symptoms. MIS-C cases were typically observed 3-6 weeks after the peak incidence of COVID-19 in the general population (4). The peak age for MIS-C was 9-10 years (3). MIS-C affects $0.5-3.1 \%$ of all diagnosed and $0.9-7.6 \%$ of all hospitalized pediatric COVID-19 patients according to a large international cohort study (18). Coronary artery dilatation or aneurysms occurred in $15-25 \%$ of cases (3). Patients frequently presented with shock or hemodynamic instability: $60-80 \%$ required hospitalization in an ICU, and 50\% required inotropes and/ or fluid resuscitation (4). In addition to supportive care, intravenous immunoglobulin (IVIG) and corticosteroids -either alone or in combination- were effective for children with MIS-C (3). In the short to medium-term, the prognoses were promising, with low rates of coronary artery aneurysms and even lower mortality (4). COVID-19 vaccines should be delayed for children with a previous history of MIS-C until clinical recovery has been achieved or 90 days after diagnosis (19). Children who received a $1-2 \mathrm{~g} / \mathrm{kg}$ dose of IVIG for the treatment of MIS-C could receive their live vaccines (MMR, varicella) at least 11 months after their treatment. Another significant post-infectious manifestation associated with SARS-CoV-2 is "long COVID" in children. Long COVID, defined by the persistence of symptoms for more than 3 months, is more common in people aged 12 and older. This condition causes a wide range of symptoms such as fatigue, shortness of breath, and impairs the daily life activities of the patients (3). The risk of long COVID is lower in children compared to other age groups (20). Existing evidence is highly heterogeneous, resulting in a wide range of prevalence estimates as $0 \%$ to $27 \%(3)$.

In addition to the direct effects of SARS-CoV-2 on children, children have suffered some of the most severe indirect effects of the pandemic. These include the impact on mental health and wellbeing, school closures, with large disruptions to in-person school, limited peer interactions, increased body mass index, delayed routine well-care visits and routine immunizations, delayed health-seeking behaviors, more prone to child abuse and neglect, and increased cyberbullying $(5,9,10,21)$. Some children have lost their parents, relatives, or caregivers.

\section{COVID-19 Vaccines in Children and Adolescents}

Severe illness from acute COVID-19 is uncommon in healthy children; however, it can occur, especially in those with pre-existing conditions. Children and adolescents play an important role in SARS-CoV-2 transmission $(3,4,5,10,13,16)$. With widespread adult immunization, younger people account for a greater proportion of COVID-19 infections. The pandemic has disrupted the education of students due to long period of school closures. As a result, the direct benefits of vaccination to the individual is lower compared to that in other age groups: However, immunization of children and adolescents with an effective and safe vaccine is likely to promote disease prevention directly and stop the spread of the infection, thereby reducing the disease burden. Vaccinating children and adolescents will facilitate their reintegration into schools and the society $(4,5,8,10,16,22)$.

As of December 2021, a limited number of COVID-19 vaccines had been approved for children and adolescents. There is limited evidence on the efficacy and safety of COVID-19 vaccines in children and adolescents. This article does not contain arguments for or against vaccinating children. COVID-19 vaccines have been approved for use in children and/or adolescents in a number of countries, including CoronaVac (Sinovac), BNT162b2 (Comirnaty; PfizerBioNTech) and mRNA1273 (Spikevax; Moderna). In most countries, primary vaccination would be recommended and started in children and adolescents regardless of underlying medical conditions. Those with a known current SARSCoV-2 infection should postpone vaccination at least until their recovery. Serologic testing for previous infection is not recommended (4).

\section{mRNA-based Vaccine Developed by Pfizer and Biontech (BNT162B2; Comirnaty)}

The Pfizer-BioNTech vaccine, BNT162b2, is made up of a nucleoside-modified mRNA molecule encoding the SARSCoV-2 spike glycoprotein in a prefusion state, enveloped within a lipoprotein nanoparticle (5). Two doses of BNT162b2 elicited high neutralizing titers and robust T-cell responses against SARS-CoV-2 in healthy adults. A double-blind randomized controlled phase III trial of over 43,000 people aged 16 and above who received either a 2-dose BNT162b2 vaccine (30 g doses) or a placebo showed a 95\% [95\% confidence interval (Cl) 90.3-97.6; 8 cases in BNT162b2 group versus 162 cases in placebo] prevention rate of symptomatic COVID-19 from seven days after dose 2 (23). BNT162b2 had a favorably safe profile in phase 2 trials involving 16-18-year-old adolescents $(22,23)$. The side effects are mild to moderate pain at the injection site, fever, fatigue, and headache. In a six-month 
follow-up report, the vaccine efficacy against symptomatic disease remained high, but slightly decreased from 96\% to 84\% between four and six months after administration (24). According to studies, plasma from individuals vaccinated with BNT162b2 retain the neutralizing activity of the vaccine against concerned variants, though the levels of neutralizing antibodies generated are lower against Beta (B.1.351), Delta (B.1.617.2) and Omicron (B.1.1.529) $(25,26)$. The vaccine is also effective against Delta variants especially in severe patients requiring hospitalization (27).

On December 2020, BNT162b2 vaccine was authorized by the Food and Drug Administration (FDA) for emergency use in the prevention of disease in people aged 16 and older (28). BNT162b2 is approved for use in 118 countries (7). The vaccine was expanded to 12-to-15-year-old individuals in May 2021, and to 5-to-11-year-old individuals on October 29, 2021 (28). The primary series of Pfizer-BioNTech COVID-19 vaccine is two intramuscular doses given three weeks (21 days) apart to all age groups. Individuals with certain immunocompromizing conditions are given a $3^{\text {rd }}$ dose at least 28 days after the second dose. A booster dose, defined as one dose given at least six months after the last dose in the primary series, is recommended for all people aged 16 and older. The only contraindications to vaccination are allergic reactions to the vaccines or their components (29).

Children aged between 12-15 years old: The immunogenicity, safety, and efficacy of the BNT162b2 vaccine were assessed in a phase 3 trial involving 2,260 adolescents aged 12 to 15 years (22). Its immunogenicity in 12-15-year-old adolescents was not inferior to that in 16-25-year-old young adults. Vaccine efficacy against symptomatic infection was $100 \%$ seven days after the second dose (95\% Cl; 78.1-100). After two doses, the vaccine efficacy was also higher among 1,983 adolescents aged 12 to 15 years without evidence of previous SARS-CoV-2 infection; vaccine efficacy was 100\%; seven or more days after dose 2 (95\% Cl 75.3-100; no cases in BNT162b2 recipients against 16 cases among placebo group). In this age group, there were no cases of severe COVID-19 infection (22). The EUA of BNT162b2 vaccine was expanded to include 12 to 16-year-old individuals by May 2021. In a study of 464 people aged 12 to 18 who were hospitalized in the United States during the summer of 2021, the vaccine effectiveness against COVID-19-related hospitalization was estimated to be $93 \%$ (14).

Children aged between 5-11 years old: The PfizerBioNTech BNT162b2 vaccine is being tested in a randomized, placebo-controlled phase trials in healthy children (28). A dose of ten mcg was chosen for the phase 2 and 3 trials in
5-11 year-old children, based on the reactogenicity observed in the initial cohort of the phase 1 trial. Thus, 4,647 children (48.6\% female, $20 \%$ had an underlying comorbidity) aged 5 to 11 were given either two doses of the vaccine or a placebo 21 days apart in the United States, Finland, Poland, and Spain. Approximately $10 \%$ of participants were seropositive SARSCoV-2, at baseline infection. BNT162b2 vaccine was safe, well tolerated, and induced robust neutralizing antibodies. Compared with neutralizing antibody titers induced in recipients aged 16 to 25 years, those induced in recipients aged 5 to 11 years with a lower dose of the vaccine were similar. The efficacy of a lower vaccine dose (given in a two-dose series) was $90.7 \%$ among 2,186 children aged 5 to 11 years with no evidence of prior infection $(95 \% \mathrm{Cl}$; 68-98; three cases among 1,305 vaccine recipients versus 16 cases among 663 placebo recipients) (28). The preliminary data did not include vaccine efficacy against hospitalization, MIS-C, or death. In the United States, COVID vaccination among 5-11 year-old individuals is expected to accelerate the decline in numbers (expected 8\%; 600,000 cases) from November 2021 to March 2022 (15).

The primary series for children aged 5 to 11 years constitutes of two intramuscular doses of $0.1 \mathrm{~mL}(10 \mathrm{mcg})$ given three weeks apart. In Canada, BNT162b2 vaccine (10 mcg) could be given to children aged 5 to 11 years with an interval of at least 8 weeks. Those who turn 12 after the first dose of the series should finish it with the dose recommended for adolescents of 12 years of age; however, if they received the lower dose after turning 12, no need for it to be repeated. BNT162b2 vaccine for children aged 5 to 11 years should not be given concurrently with other live or inactivated vaccines. It is best to wait at least 14 days before or after administering other vaccines (11).

There are some differences between the adult/adolescent (12 years and older) and pediatric (5-11 years) formulations of the Pfizer-BioNTech COVID-19 vaccine. The adult and adolescent formulations have purple vial caps, while pediatric formulations have orange vial caps. The diluent volume is 1.8 $\mathrm{mL}$ for adult/adolescent formulation, the dose is $0.3 \mathrm{~mL}$ ( 30 $\mathrm{mcg}$ ), and there are six doses per vial. However, the diluent volume is $1.3 \mathrm{~mL}$ for pediatric formulation, the dose is $0.2 \mathrm{~mL}$ (10 mcg), and there are ten doses per vial. The post-dilution time is 6 hours at room temperature for the adult/adolescent formulation and 12 hours for the pediatric formulation (30).

\section{The Safety of BNT162b2 Vaccine in Children and Adolescents}

Children and adolescents receiving BNT162b2 vaccine reported more local and systemic events (generally mild to moderate severity) in both cohorts (5-11 and 12-15 years 
old). These local and systemic events typically resolved within one or two days. Injection site pain, fatigue, headache, and fever were common in 12-15 year-old, as they were in young adults and adults. Fever occurred after second dose in $20 \%$ of 12-to-15-year-old individuals and in 17\% of 16-to-25 year-old individuals. Severe adverse events were reported in $0.6 \%$ in children aged 12 to 15 years and $1.7 \%$ of those aged 16 to 25 years. During these trials, there were no serious adverse events related to the vaccine (cases of myo/pericarditis, MIS-C, or deaths). After routine use of vaccine, myocarditis/ pericarditis have been rarely reported (detailed discussed in later part) $(11,22,28)$.

\section{Moderna mRNA-based Vaccine (mRNA1273; Spikevax)}

Moderna mRNA-based vaccine (mRNA-1273), is made up of a nucleoside-modified mRNA molecule encapsulated within a lipoprotein nanoparticle (4). This double-blind placebo-controlled study with over 30,000 adults (two doses given 28 days apart) demonstrated a 94.1\% efficacy in preventing COVID-19 infection, including severe COVID-19 disease (31). After an average of 5.2 months, the vaccine efficacy was still at $93.2 \%$ for symptomatic infection and 98.2\% for severe disease (32). During the clinical trials, no safety concerns were identified. On December 18, 2020, the FDA granted the vaccine EUA for people aged 18 and older. A recent phase 3 clinical trial (with 3,700 participants) with mRNA1273 vaccine found that it could be well tolerated and effective in children and adolescents aged 12 to 17 years old (not published, 33). Immunogenicity in adolescents aged 12 to 17 years is comparable to or greater than that seen in young adults. European Medicines Agency's (EMA) human medicines committee has recommended granting an extension of indication for the mRNA1273 to be used equally in children aged 12 to 17 years in Europe (33). Vaccination with the mRNA1273 vaccine for children as young as 12 years old is now recommended in some countries. These vaccines are linked to a rare risk of myocarditis (which occurs more frequently in adolescents and young adults) (34).

\section{Inactivated SARS-COV-2 Vaccine (CoronaVac, Sinovac)}

CoronaVac, is an inactivated SARS-CoV-2 vaccine and clinical trials revealed that this vaccine was safe, immunogenic and effective in adults aged 18 and older $(35,36)$. According to a phase III trial with 10,000 participants in Turkey, the vaccine efficacy at 14 days after full vaccination was $83.5 \%(95 \% \mathrm{Cl}$
65.4-92.1) (37). In a Chilean observational study involving over 10 million people, the vaccine effectiveness was estimated at $70 \%$ in preventing COVID-19 and $86-88 \%$ in preventing hospitalization or deaths (38). A subsequent study in Brazil demonstrated lower vaccine effectiveness among adults over the age of 70 in the context of the prevalent Gamma variant (47\%, 56\%, and $61 \%$ against COVID-19, hospitalizations, and deaths, respectively) (39). Since the end of 2020, CoronaVac has been one of the most widely used vaccines among adults worldwide. This vaccine is available in China and a few other countries, including Brazil, Chile, Indonesia, Mexico, and Turkey (7).

In a double-blind, randomized, controlled, phase 1/2 clinical trial in China, Han et al. (40) assessed the safety, tolerability, and immunogenicity of an inactivated SARS-CoV-2 vaccine (CoronaVac) in healthy children and adolescents aged 3 to 17 years. Between October and December 2020, 72 participants were enrolled in the phase 1 study, while 480 participants were enrolled in phase 2 . This vaccine was safe and well tolerated, and it elicited humoral responses. After two doses of vaccination, the seroconversion rates of neutralizing antibodies were greater than $96 \%$, and the neutralizing antibody titers induced by the $3.0 \mu \mathrm{g}$ dose were higher than those induced by the $1.5 \mu \mathrm{g}$ dose. The most common reactions were injection site pain (13\%), followed by fever (5\%). The limitations of this study include the lack of assessment of T-cell responses and the fact that all participants were recruited from a single country, China (40). Furthermore, this is a phase 1-2 study, and there is no prior evidence on the vaccine efficacy/effectiveness. China, Chile, and Turkey have been using this vaccine with an emergency authorization in children and adolescents; the effectiveness of the vaccines from these countries will highlight their effect on COVID-19 epidemiology in these age groups. This vaccine has not yet received authorization for emergency use in children form the World Health Organization (WHO) (9).

\section{Other Pediatric COVID-19 Vaccines}

Another inactivated vaccine, BBIBP-CorV (Sinopharm), was approved for children and adolescents aged between 3 to 17 years in China. Covaxin is another adjuvant inactivated vaccine developed by Bharat in India, and was approved in India for 12-17-year-old individuals. ZycovD, a novel DNA vaccine, has been approved by the Indian regulatory authorities for the same age group. These three vaccines not yet received authorization from the WHO emergency use listing procedure (9). 


\section{Routine use of COVID-19 Vaccines Among Children/ Adolescents and Real-world Effectiveness Data}

Pfizer-BioNTech BNT162b vaccine is one of the most commonly used vaccine among children and adolescents. There is only one study about the real-world effectiveness of pediatric COVID-19 vaccines. Following the introduction of the BNT162b vaccine in United States, among 68 hospitalized adolescents; four were fully and five were partially vaccinated while 59 were unvaccinated. The hospitalization rate among unvaccinated adolescents was ten times higher than that of fully vaccinated adolescents, indicating that vaccines were highly effective at preventing serious COVID-19 illness during a period when the Delta variant predominated (14).

The EMA approved the Pfizer-BioNTech BNT162b vaccine for children aged 12-15 in May 2021 and for children aged 5-11 in November 2021, making it the first COVID-19 vaccine to receive such approval in the European Union (41). The EMA also approved the Moderna mRNA1273 vaccine for use in children aged 12 to 17 in July 2021 (33). A majority of European countries initiated the routine use of these vaccines in children and adolescents. In England, all adolescents aged 16 and 17 years started receiving vaccines on August 23, 2021, followed by 12-15 year-old individuals in September. By the December 2021, approximately 1.3 million children aged 12 to 15 had received at least one dose. Initially, this age group was only given one dose, but due to concerns about the Omicron variant, a second dose has been recently introduced (only 29,000 children are fully vaccinated) (42). In Scotland, 59\% of children have received at least one dose of the mRNA vaccine, as well as $54 \%$ in Wales and $>60 \%$ in Ireland (43). In Italy, $75.5 \%$ of $12-19$ year-old individuals had received at least one dose as of December 17, 2021, with $71.4 \%$ fully vaccinated. Italy recently approved the Pfizer-BioNTech BNT162b vaccine for children aged 5 to 11 years, and 28,000 children received their first dose already (44). In Germany, on August 16, 2021, STIKO decided to vaccinate all children over the age of 12 . As of December 2021, 50\% of children aged 12-17 years old had received both doses (45). In France, 70\% of children aged 12 to 17 years old have received at least one dose of mRNA vaccine, and $77 \%$ are fully vaccinated (46). In Spain, as of December 17 , 2021, 88.8\% of children and adolescents aged 12 to 19 years old had received their first dose of mRNA vaccine, and $85.5 \%$ were fully vaccinated. The health authorities of Spain will initiate the vaccination of children aged 5 to 11 in December 2021 (47). In Turkey, both the Pfizer-BioNTech mRNA vaccine and the inactivated vaccine (CoronaVac) are approved for use in children over the age of 12 . CoronaVac vaccine is routinely used in Chile in children over the age of six.

\section{The Benefits and Risks of Pediatric COVID-19 Immunization Strategies}

The WHO published a statement on COVID-19 vaccination for children and adolescents at the end of November 2021. When developing COVID-19 immunization policies and programs, vaccinating children and adolescents has advantages that go beyond direct and indirect health benefits (9).

When comparing adults and the elderly population, the rates of hospitalization, ICU admissions, and mortality are quite low $(3,4,5)$; therefore, routine pediatric COVID-19 immunization is not as compelling as it is for adults. However, children are at risk for severe COVID-19, MIS-C, and long COVID (10). A SARS-CoV-2 vaccine for children and adolescents will be critical in containing the COVID-19 pandemic. Children and adolescents also play an important role in the spread of SARSCoV-2 in communities (10). High vaccination rates in adults in the community must be encouraged to provide indirect protection to children. However, adult vaccination coverage fell short of the herd immunity threshold in most parts of the world. There are safe and effective vaccines that have been approved for pediatric use by health-care authorities. As a result, parents willing to immunize their children must have access to these vaccines. In addition, children at high risk of severe COVID-19 or those with specific conditions such as those with neurological cardiac, respiratory, and renal diseases, Down syndrome, immunodeficiencies, malignancies, obesity, and diabetes (13), should be prioritized for vaccination. Routine immunization would be beneficial in low/middleincome countries due to an increase in the number of cases with co-morbidities. In high-income countries, routine immunization for deprived and ethnic minority groups could equally be beneficial, because of severe disease and MIS-C are common in these groups (10).

In the pediatric age group, the risk-benefit balance of vaccination is more complicated (10). Zimmermann et al. (10) summarized the pros and cons of pediatric COVID-19 immunization. Considerations for vaccination in children include potential vaccine protection against COVID-19, severe COVID-19 infection, MIS-C, and long COVID; potential contribution to reducing community transmission, and potential impact against the spread of new variants. Widespread pediatric immunization is associated with avoiding isolation, quarantine, lockdown, and school closure, resulting in a faster return to pre-pandemic activity and economic stability. However, for the evidence backing up these potential benefits is very limited. Potential reasons for non-routine vaccination of children include a generally mild 
course of disease during childhood, adverse events associated with vaccines, unknown efficacy against MIS-C or long COVID, and an unknown efficacy against SARS-CoV-2 transmission. Concerns about routine COVID-19 immunizations include vaccine cost, supply issues, and co-administration with other childhood vaccines (10). If health authorities decide to include the vaccine in routine immunization, the risks and benefits must be reevaluated on a regular basis as new variants could emerge, as well as new findings on its efficacy and adverse effects $(9,10)$.

\section{MIS-C and Long COVID}

Multisystemic inflammatory syndrome in children is an important end-point for potential pediatric/adolescent immunization. The risk of MIS-C and overall mortality from MIS-C are low, but the majority of patients required ICU admission $(4,7,10)$. The long-term consequences of MIS-C are unknown. Owing to its serious signs and complications requiring ICU, the cost of hospital stay, the requirement for IVIG-steroid and other biological treatment, and the fear and anxiety of MIS-C results in an important end-point for COVID-19 vaccine real-world effectiveness. To date, no evidence on vaccine efficacy/effectiveness against MIS-C is available. Since the pathogenesis of MIS-C is unknown, there is a theoretical risk that COVID-19 vaccination will cause MIS-C. By the end of October 2021, according to the EMA report, no substantial evidence on a possible link between mRNA COVID-19 vaccines and MIS-C have been demonstrated (48). Long COVID is another potential important end-point for COVID-19 vaccines in children and adolescents. There is little or no evidence on vaccine efficacy or real-world effectiveness against Long COVID.

\section{The Safety of Pediatric/Adolescent COVID-19 Immunization}

The main question for implementing any vaccine is "do the benefits of the vaccine in preventing disease outweigh any known or potential risks associated with vaccination?" (10). Many countries have authorized the use of previously discussed COVID-19 vaccines for EUA in children and adolescents, and millions of children and adolescents have been vaccinated worldwide. Vaccines are well tolerated for children and adolescents except for effects like pain the injection site, fever, and fatigue. Following the routine use of mRNA vaccines, there have been some cases of myocarditis/ pericarditis among adolescents and young adults, particularly among males (8). Following the second dose, Schauer et al. (49) estimated a $0.008 \%$ incidence of myopericarditis in adolescents aged $16-17$ years and a $0.01 \%$ incidence in those aged 12 to 15 years. The majority of cases with myocarditis and pericarditis were mild and self-limiting, and recovered without complications (8). After receiving an mRNA vaccine, adolescents, and young adults who experienced new chest pain, palpitation, shortness of breath were reevaluated for myocarditis. Other causes of myocarditis should also be considered (50). Moreover, the risk of myocarditis associated with COVID-19 infection is higher in this age group, and there is limited information about the risk and long-term outcome of myocarditis/pericarditis due to acute COVID-19 infection in children and adolescents. As of December 12, 2021, the Pfizer-BioNTech vaccine had been administered to 7.1 million children in in the United States. The majority of reactions reported were injection site-related, mild to moderate in severity, most frequently reported the day after vaccination, and slightly more frequently reported after the second dose. Missing school was rarely reported, and only $1 \%$ sought medical attention. According to VAERS reports, eight cases with myocarditis (four girls and four boys, two after first dose and six after second dose) have been detected among children aged 5 to 11 years (51). As a precaution, the second dose of the mRNA COVID-19 vaccination series should be postponed in children who experience myocarditis or pericarditis following the first dose (11). As a result, it was determined that the benefit of COVID-19 vaccination for adolescents, outweighed the risk of peri/myocarditis associated with mRNA vaccination. In October 2021, the WHO Global Advisory Committee on Vaccine Safety also concluded that the benefits of mRNA COVID-19 vaccines outweighed the risks in all age groups (9). The definition, reporting, and long-term follow-up of myocarditis/pericarditis cases are critical.

\section{Transmission and New Variants}

Another potential benefit of immunizing children is that it helps to reduce transmission, thereby reducing severe cases in adults and the risk of emergence of new variants. For the Delta variant, it has been suggested that infected fully vaccinated adults are just as likely to transmit SARS-CoV-2 as infected unvaccinated individuals, within a shorter period of time. While transmission from children is quite low in educational settings, infants, and children may be the index case for household transmission. There are conflicting findings regarding the effectiveness in preventing virus transmission, particularly during the Delta variant's predominance season (10).

Several SARS-CoV-2 variants have been identified because of their potential for immune escape. Based on data from efficacy trials and immunogenicity studies, COVID-19 vaccines likely remain effective against the variants, but efficacy 
may attenuate against Delta (B.1.617.2) and Beta (B.1.351) (27). Omicron (B.1.1.529) is a newly defined variant and preliminary reports suggest that neutralizing activity of sera from vaccinated individuals is lower against the Omicron variant compared to the wild-type virus (17). However, previously infected individuals who are vaccinated and individuals who receive booster vaccination retain adequate neutralizing titers against Omicron. In a population with a low number of vaccinated adults, infected children spread the variant of concern. Therefore, this is also stronger argument for vaccinating children who live with high risk household members.

\section{Booster Dose}

Booster dose has been recommended for adolescents over the age of 12 , and there is no information on booster dose requirements/schemes for children under the age of 12 .

\section{Vaccine Supply, Vaccine Inequity, and Vaccine Hesitancy}

Another important factor to consider is the limited global supply of COVID-19 vaccine. Vaccines are now best way to return to a normal life scenario, if equally distributed worldwide. To date, many low/middle-income countries have only been able to vaccinate less than $5 \%$ of their population. Available vaccines may be better prioritized for vaccinating adults with a higher risk of severe COVID-19, such as healthcare workers. However, some countries start to immunize their population with $3^{\text {rd }}$ or $4^{\text {th }}$ booster dose or expanded their age coverage with children; many lower-middle-income countries still lack sufficient vaccine supply to offer a primary vaccination series to their highest priority-use groups. If this vaccine inequity does not end soon, it is likely that poorer countries will be left behind once again losing thousands of vaccine-preventable lives and leading to more virulent virus variants like Omicron to appear $(10,52)$.

Vaccine hesitancy was an emerging concern for routine immunization prior to the pandemic, and it unfortunately increased during the pandemic period (21). Mandatory
COVID-19 vaccination of children has been proposed in order to achieve population-level vaccination coverage and herd immunity. There is insufficient data to make a definitive decision on whether the COVID-19 vaccine should be made mandatory for children. Children, adolescents, and their parents should be supported and respected in their decisions regarding vaccinations for their children, regardless of the decisions they make (4).

Global health authorities, including WHO, EMEA, CDC, and other national health authorities have already authorized the emergency use of COVID-19 vaccines in children and adolescents. The WHO global vaccination strategy targets are $40 \%$ of each country's population by the end of 2021 , and $70 \%$ for 2022 and to date, these targets have not yet been achieved (9). Increasing vaccine coverage among adults and the elderly is still beneficial for controlling pediatric disease burden, but the herd immunity threshold is still lower than expected. Although benefit-risk assessments clearly support the benefit of vaccinating all age groups, the direct health benefit of vaccinating children and adolescents is lower. Clinical trials of inactivated vaccines and mRNA vaccines in children and adolescents have revealed a favorable safety profile as well as immunogenicity. There are also promising results of mRNA vaccine's efficacy and real-world effectiveness. Vaccinating children is likely to provide a direct benefit of disease prevention as well as indirect benefits such as community protection. This benefit would be enhanced for vulnerable children who are at high risk of severe COVID-19. Short and long-term complications related with COVID-19 infection could benefit from pediatric vaccination. Vaccination may also help to reduce school closures and quarantine requirements $(5,8,9,10)$. Global and national health authorities should also closely monitor and constantly assess the benefits and potential risks of vaccination in children and adolescents.

\section{Ethics}

Peer-review: Externally peer-reviewed.

Financial Disclosure: The author declared that this study received no financial support. 


\section{REFERENCES}

1. COVID-19 Dashboard. Available from: https://coronavirus.jhu.edu/ map.html. Accessed December 17, 2021.

2. Kompaniyets L, Pennington AF, Goodman $A B$, et al. Underlying medical conditions and severe illness among 540,667 adults hospitalized with COVID-19, March 2020-March 2021. Prev Chronic Dis 2021;18:E66.

3. Howard-Jones AR, Burgner DP, Crawford NW, et al. COVID-19 in children. II: pathogenesis, disease spectrum and management. J Paediatr Child Health 2021;10.1111/jpc.15811.

4. Blanchard-Rohner G, Didierlaurent A, Tilmanne A, Smeesters P, Marchant A. Pediatric COVID-19: immunopathogenesis, transmission and prevention. Vaccines (Basel) 2021;9:1002.

5. Galindo R, Chow H, Rongkavilit C. COVID-19 in children: clinical manifestations and pharmacologic interventions including vaccine trials. Pediatr Clin North Am 2021;68:961-976

6. Krammer F. SARS-CoV-2 vaccines in development. Nature 2020;586:516-527.

7. CovID-19 Vaccine Tracker. Available from: https://covid19. trackvaccines.org/. Accessed December 17, 2021.

8. Lv M, Luo X, Shen Q, et al. Safety, immunogenicity, and efficacy of COVID-19 vaccines in children and adolescents: a systematic review. Vaccines (Basel) 2021;9:1102.

9. Interim statement on COVID-19 vaccination for children and adolescents. Available from: https://www.who.int/news/item/24-112021-interim-statement-on-covid-19-vaccination-for-children-andadolescents. Accessed December 17, 2021.

10. Zimmermann P, Pittet LF, Finn A, Pollard AJ, Curtis N. Should children be vaccinated against COVID-19? Arch Dis Child 2021; archdischild-2021-323040.

11. National Advisory Committee on Immunization (NACl) statement: Recommendation on the use of the Pfizer-BioNTech COVID-19 vaccine $(10 \mathrm{mcg})$ in children 5 to 11 years of age. Available from: https://www.canada.ca/en/public-health/services/immunization/ national-advisory-committee-on-immunization-naci/ recommendations-use-covid-19-vaccines/pfizer-biontech-10-mcgchildren-5-11-years-age.html. Accessed December 17, 2021.

12. Molteni E, Sudre $\mathrm{CH}$, Canas LS, et al. Illness characteristics of COVID-19 in children infected with the SARS-CoV-2 Delta variant. medRxiv 2021.10.06.21264467.

13. Graff K, Smith C, Silveira L, et al. Risk factors for severe COVID-19 in children. Pediatr Infect Dis J 2021;40:e137-e145.

14. Delahoy MJ, Ujamaa D, Whitaker M, et al. Hospitalizations associated with COVID-19 among children and adolescents - COVID-NET, 14 states, March 1, 2020-August 14, 2021. MMWR 2021;70:1255-1260.

15. EtR Framework: Pfizer-BioNTech COVID-19 vaccine in children aged 5-11 years. Available from: https://www.cdc.gov/vaccines/acip/ meetings/downloads/slides-2021-11-2-3/08-COVID-Oliver-508.pdf. Accessed December 17, 2021.
16. Howard-Jones AR, Bowen AC, Danchin M, et al. COVID-19 in children: I. Epidemiology, prevention and indirect impacts. J Paediatr Child Health 2021;10.1111/jpc.15791.

17. Update on Omicron variant. Available from: https://www.cdc.gov/ vaccines/acip/meetings/downloads/slides-2021-12-16/06-COVIDScobie-508.pdf. Accessed December 17, 2021.

18. Duarte-Salles T, Vizcaya D, Pistillo A, et al. Thirty-day outcomes of children and adolescents with COVID-19: an international experience. Pediatrics 2021;148:e2020042929.

19. Multisystem imflamamatory syndrome in children (MIS-C) interim Guidanca. Available from: https://www.aap.org/en/pages/2019novel-coronavirus-covid-19-infections/clinical-guidance/ multisystem-inflammatory-syndrome-in-children-mis-c-interimguidance/. Accessed December 17, 2021.

20. Radtke T, Ulyte A, Puhan MA, Kriemler S. Long-term symptoms after SARS-CoV-2 infection in children and adolescents. JAMA 2021;326:869-871.

21. Dinleyici EC, Borrow R, Safadi MAP, van Damme P, Munoz FM. Vaccines and routine immunization strategies during the COVID-19 pandemic. Hum Vaccin Immunother 2021;17:400-407.

22. Frenck RW Jr, Klein NP, Kitchin N, et al. Safety, immunogenicity, and efficacy of the BNT162b2 Covid-19 vaccine in adolescents. N Engl J Med 2021;385:239-250.

23. Polack FP, Thomas SJ, Kitchin N, et al. Safety and efficacy of the BNT162b2 mRNA Covid-19 vaccine. N Engl J Med 2020;383:26032615.

24. Thomas SJ, Moreira ED Jr, Kitchin N, et al. Safety and efficacy of the BNT162b2 mRNA Covid-19 vaccine through 6 months. N Engl J Med 2021;385:1761-1773.

25. Falsey AR, Frenck RW Jr, Walsh EE, et al. SARS-CoV-2 Neutralization with BNT162b2 Vaccine Dose 3. N Engl J Med 2021;385:1627-1629.

26. Lopez Bernal J, Andrews N, Gower C, et al. Effectiveness of Covid-19 vaccines against the B.1.617.2 (Delta) variant. $N$ Engl J Med 2021;385:585-594.

27. Lu L, Mok BW, Chen LL, et al. Neutralization of SARS-CoV-2 Omicron variant by sera from BNT162b2 or Coronavac vaccine recipients. Clin Infect Dis 2021;ciab1041.

28. Walter EB, Talaat KR, Sabharwal C, et al. Evaluation of the BNT162b2 Covid-19 vaccine in children 5 to 11 years of age. $\mathrm{N}$ Engl J Med 2021;NEJMoa2116298.

29. https://www.cdc.gov/coronavirus/2019-ncov/vaccines/differentvaccines/Pfizer-BioNTech.html. Accessed December 17, 2021.

30. https://www.cdc.gov/vaccines/covid-19/downloads/Pfizer-PediatricReference-Planning.pdf. Accessed December 17, 2021.

31. Baden LR, El Sahly HM, Essink B, et al. Efficacy and safety of the mRNA-1273 SARS-CoV-2 vaccine. N Engl J Med 2021;384:403-416.

32. El Sahly HM, Baden LR, Essink B, et al. Efficacy of the mRNA-1273 SARS-CoV-2 vaccine at completion of blinded phase. N Engl J Med 2021;385:1774-1785. 
33. Spikevax (previously COVID-19 Vaccine Moderna). https://www. ema.europa.eu/en/medicines/human/EPAR/spikevax. Accessed December 17, 2021.

34. Patone M, Mei XW, Handunnetthi L, et al. Risks of myocarditis, pericarditis, and cardiac arrhythmias associated with COVID-19 vaccination or SARS-CoV-2 infection. Nat Med 2021. doi: 10.1038/ s41591-021-01630-0.

35. Zhang Y, Zeng G, Pan H, et al. Safety, tolerability, and immunogenicity of an inactivated SARS-CoV-2 vaccine in healthy adults aged 18-59 years: a randomised, double-blind, placebo-controlled, phase 1/2 clinical trial. Lancet Infect Dis 2021;21:181-192.

36. Wu Z, Hu Y, Xu M, et al. Safety, tolerability, and immunogenicity of an inactivated SARS-CoV-2 vaccine (CoronaVac) in healthy adults aged 60 years and older: a randomised, double-blind, placebocontrolled, phase 1/2 clinical trial. Lancet Infect Dis 2021;21:803812.

37. Tanriover MD, Doğanay HL, Akova M, et al. Efficacy and safety of an inactivated whole-virion SARS-CoV-2 vaccine (CoronaVac): interim results of a double-blind, randomised, placebo-controlled, phase 3 trial in Turkey. Lancet 2021;398:213-222.

38. Bueno SM, Abarca K, González PA, et al. Safety and Immunogenicity of an Inactivated SARS-CoV-2 vaccine in a subgroup of healthy adults in chile. Clin Infect Dis 2021:ciab823.

39. Ranzani OT, Hitchings MDT, Dorion M, et al. Effectiveness of the CoronaVac vaccine in older adults during a gamma variant associated epidemic of covid-19 in Brazil: test negative case-control study. BMJ 2021;374:n2015.

40. Han B, Song Y, Li C, et al. Safety, tolerability, and immunogenicity of an inactivated SARS-CoV-2 vaccine (CoronaVac) in healthy children and adolescents: a double-blind, randomised, controlled, phase 1/2 clinical trial. Lancet Infect Dis 2021;21:1645-1653.

41. https://www.ema.europa.eu/en/news/comirnaty-covid-19vaccine-ema-recommends-approval-children-aged-5-11. Accessed December 17, 2021.
42. https://www.england.nhs.uk/statistics/statistical-work-areas/covid19-vaccinations/. Accessed December 17, 2021.

43. http://partenaire.euronews.net/next/2021/12/06/covid-vaccine-forchildren-who-in-europe-is-leading-the-race. Accessed December 17, 2021.

44. https://www.governo.it/it/cscovid19/report-vaccini/.Accessed December 17, 2021.

45. https://impfdashboard.de/en/. Accessed December 17, 2021.

46. https://solidarites-sante.gouv.fr/grands-dossiers/vaccin-covid-19/ article/le-tableau-de-bord-de-la-vaccination. Accessed December 17, 2021.

47. https://www.mscbs.gob.es/profesionales/saludPublica/ccayes/ alertasActual/nCov/pbiVacunacion.htm. Accessed December 17, 2021.

48. European Medicines Agency (EMA). Meeting highlights from the Pharmacovigilance Risk Assessment Committee (PRAC) 25-28 October 2021. Available from: https://www.ema.europa.eu/en/ news/meeting-highlisgts-pharmacovigilance-risk-assessmentcommitee-prac-25-28-october-2021. Accessed December 17, 2021.

49. Schauer J, Buddhe S, Colyer J, et al. Myopericarditis after the Pfizer messenger ribonucleic acid coronavirus disease vaccine in adolescents. J Pediatr 2021;238:317-320.

50. Clinical considerations: myocarditis and pericarditis after Receipt of mRNA COVID-19 vaccines among adolescents and young adults. Available from: https://www.cdc.gov/vaccines/covid-19/clinicalconsiderations/myocarditis.html. Accessed December 17, 2021.

51. Adverse events among children ages 5-11 years after COVID-19 vaccination: updates from v-safe and the Vaccine Adverse Event Reporting System (VAERS). Available from: https://www.cdc.gov/ vaccines/acip/meetings/downloads/slides-2021-12-16/05-COVIDSu-508.pdf. Accessed December 17, 2021.

52. Lanziotti VS, Bulut Y, Buonsenso D, Gonzalez-Dambrauskas S. Vaccine apartheid: this is not the way to end the pandemic. J Paediatr Child Health 2021;10.1111/jpc.15805. 